\title{
Artificial oxidative stress-tolerant Corynebacterium glutamicum
}

\author{
Joo-Young Lee ${ }^{1}$, Hyo Jung Lee ${ }^{1}$, Jiyoon Seo ${ }^{1,4}$, Eung-Soo Kim², Heung-Shick Lee ${ }^{3}$ and Pil Kim
}

\begin{abstract}
We have reported a transcription profile of an adapted Corynebacterium glutamicum that showed enhanced oxidative stress resistance. To construct an artificial oxidative stress-resistant strain, gene clusters in the $\beta$-ketoadipate pathway, which were up-regulated in the adapted strain, were artificially expressed in the wild-type $C$. glutamicum. The wild-type strain was unable to grow under $2 \mathrm{mM} \mathrm{H}_{2} \mathrm{O}_{2}$ containing minimal medium, while the strains expressing pca gene clusters restored growth under the same medium, and the pcaHGBC expression showed the most significant effect among the gene clusters. The expressions of pca gene clusters also enabled the wild-type to increase its resistance against oxidative stressors, such as diamide and cumene hydroperoxide, as well as $\mathrm{H}_{2} \mathrm{O}_{2}$. The oxidative stress tolerance of the strain was correlated to the reactive oxygen species (ROS)-scavenging activity of the cell extract. The reason for the enhanced oxidative stress-resistance of C. glutamicum and its applications on the synthetic strain development are discussed.
\end{abstract}

Keywords: Corynebacterium glutamicum; $\beta$-ketoadipate pathway; Oxidative stress-tolerance; pca gene clusters

\section{Introduction}

Corynebacterium glutamicum, a Gram-positive bacterium with high GC-content that belongs to the order of $A c$ tinomycetales, is a well-known industrial strain for the production of various amino acids and nucleotides, such as lysine, glutamate, and inosine 5-monophosphate (IMP) (Eggeling and Bott 2005). During the fermentation processes, the industrial strains encounter many artificially-driven stresses, such as temperature, $\mathrm{pH}$, osmotic pressure, starvation, and oxidation. These kinds of stressors cause the loss of viability and cellular functions, which lower the productivity of bioprocesses (Li et al. 2009). Because reactive oxygen species (ROS) such as superoxide radical, hydroxyl radical, and hydrogen peroxide are mainly formed during respiration, by the incomplete reduction of oxygen, and because oxidative stress by high oxidizing potential of ROS leads many damages, such as mutations, metabolic pathway disruption, and growth inhibition, oxidative stress is an unavoidable damage for oxygenic bioprocess of aerobic organisms (Fridovich 1998).

\footnotetext{
* Correspondence: kimp@catholic.ac.kr

'Department of Biotechnology, The Catholic University of Korea, Bucheon, Gyeonggi 420-743, Korea

Full list of author information is available at the end of the article
}

To understand the oxidative stress-induced responses of C. glutamicum, we have adaptively evolved the wild-type strain (ATCC 13032) under gradually increasing $\mathrm{H}_{2} \mathrm{O}_{2}$ conditions in a chemostat culture for $1,900 \mathrm{~h}$, and have acquired a strain that was able to grow under $10 \mathrm{mM}$ $\mathrm{H}_{2} \mathrm{O}_{2}$ conditions (Lee et al. 2013b). The $\mathrm{H}_{2} \mathrm{O}_{2}$-adapted C. glutamicum strain (KCTC12280BP, i.e., HA strain) showed a distinguished transcriptome pattern (NCBI Gene Expression Omnibus access code: GSE41232). One of the unique transcriptome pattern of the adaptedHA strain was the up-regulations of genes involved in the degradation of aromatic compounds ( $p$-coumarate, benzoate, quinate, shikimate, ferulate, vanillate, caffeate) in $\beta$-ketoadipate pathway, which could be linked to TCA cycle (Figure 1A), even though no aromatic compound was supplemented in the medium. This result brought about the theory that there might have been synthesis of aromatic antioxidants via the up-regulated $\beta$-ketoadipate pathway, and the ROS-scavenging activity of the intermediates in the $\beta$-ketoadipate pathway might have enabled the C. glutamicum HA strain to tolerate oxidative stress.

To verify this theory, we artificially expressed $p c a$ gene clusters in $\beta$-ketoadipate pathway (pcaIJ, pcaFD, and pcaHGBC) in the wild-type C. glutamicum and checked their survivability under oxidative stress conditions. The

\section{实}

(c) 2014 Lee et al.; licensee Springer. This is an Open Access article distributed under the terms of the Creative Commons Attribution License (http://creativecommons.org/licenses/by/2.0), which permits unrestricted use, distribution, and reproduction in any medium, provided the original work is properly credited. 


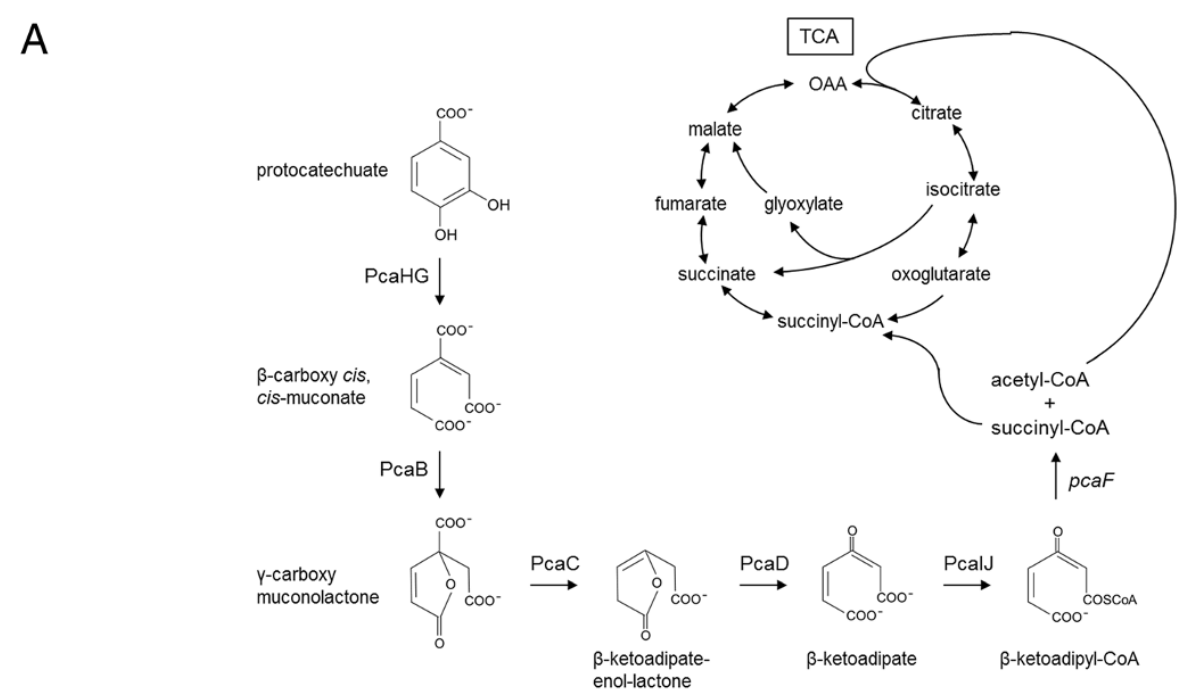

B

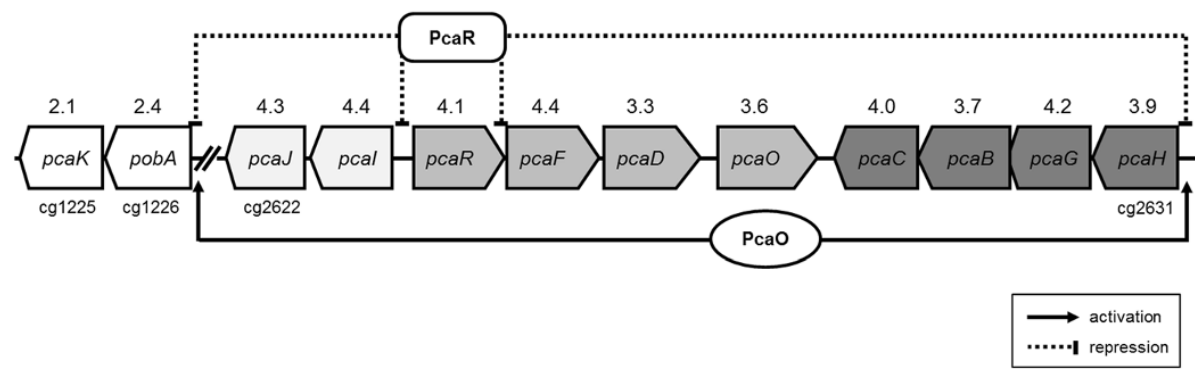

Figure 1 Schematic diagram of the $\beta$-ketoadipate pathway (A) and distribution of pca gene clusters (B) in Corynebacterium glutamicum. (A). Descriptions and abbreviations: PcaHG, PCA 3, 4-dioxygenase; PcaB, $\beta$-carboxy-cis,cis-muconate cycloisomerase; PcaC, $\gamma$-carboxymuconolactone decarboxylase; PcaD, $\beta$-ketoadipate enol-lactone hydrolase; PcalJ, $\beta$-ketoadipate:succinyl-coenzyme A transferase; PcaF, $\beta$-ketoadipate acetyl CoA acetyltransferase; TCA, tricarboxylic acid cycle. (B). Genes organized as an operon are shown in the same color. Arrows indicate transcriptional activation and repression (Shen et al. 2012; Zhao et al. 2010). Numbers above genes indicate expression ratios of $\mathrm{H}_{2} \mathrm{O}_{2}$-adapted strain/wild-type from transcriptome results using RNA-seq (NCBI Gene Expression Omnibus access code: GSE41232) (Lee et al. 2013b).

ROS-scavenging activities of the cell extracts were also estimated. Artificial oxidative stress-tolerant $C$. glutamicum is discussed based on the viewpoints of industrial applications.

\section{Materials and methods}

\section{Strain and growth condition}

Corynebacterium glutamicum ATCC 13032 containing vectors were grown in MCGC minimal medium composed of glucose $10 \mathrm{~g}$, $\left(\mathrm{NH}_{4}\right)_{2} \mathrm{SO}_{4} 4 \mathrm{~g}, \mathrm{KH}_{2} \mathrm{PO}_{4} 3 \mathrm{~g}, \mathrm{Na}_{2} \mathrm{HPO}_{4}$ $6 \mathrm{~g}, \mathrm{NaCl} 1 \mathrm{~g}$, sodium citrate dehydrate $1 \mathrm{~g}$, biotin $200 \mu \mathrm{g}$, thiamine $\cdot \mathrm{HCl} 1 \mathrm{mg}$, and minerals $\left(\mathrm{FeSO}_{4} \cdot 7 \mathrm{H}_{2} \mathrm{O} 20 \mathrm{mg}\right.$, $\mathrm{MgSO}_{4} \cdot 7 \mathrm{H}_{2} \mathrm{O} 0.2 \mathrm{~g}, \mathrm{MnSO}_{4} \cdot \mathrm{H}_{2} \mathrm{O} 2 \mathrm{mg}, \mathrm{FeCl}_{3} 2 \mathrm{mg}$, $\mathrm{ZnSO}_{4} \cdot 7 \mathrm{H}_{2} \mathrm{O} \quad 0.5 \mu \mathrm{g}, \quad \mathrm{CuCl}_{2} \cdot 2 \mathrm{H}_{2} \mathrm{O} \quad 0.2 \mu \mathrm{g}, \quad\left(\mathrm{NH}_{4}\right)_{6}$ $\mathrm{Mo}_{7} \mathrm{O}_{24} \cdot 4 \mathrm{H}_{2} \mathrm{O} \quad 0.1 \mu \mathrm{g}, \mathrm{Na}_{2} \mathrm{~B}_{4} \mathrm{O}_{7} \cdot 10 \mathrm{H}_{2} \mathrm{O} \quad 0.2 \mu \mathrm{g}$, and $\mathrm{CaCl}_{2} 70 \mu \mathrm{g}$ ) per liter (von der Osten et al. 1989). Kanamycin $(25 \mu \mathrm{g} / \mathrm{mL})$ was supplemented to maintain vectors. Hydrogen peroxide (2 $\mathrm{mM})$ was added to verify the growth against oxidative stress. Culture was performed at $30^{\circ} \mathrm{C}, 230 \mathrm{rpm}$ in a $250 \mathrm{~mL}$-Erlenmyer flask containing $50 \mathrm{~mL}$ medium. Cell growth was measured at O.D.600nm and was converted into biomass with an extinction coefficient of 0.250 .

\section{Plasmid construction}

Plasmid pSL360 (Park et al. 2004), an empty expression vector carrying the $\mathrm{P}_{180}$ promoter, which induces constitutive overexpression of the cloned gene, was used to express $p c a$ gene clusters (Figure 1B). The pca gene clusters (pcaIJ: 5' -gactgcagtgaacattacgttagcatgt-3' and $5^{\prime}$-ggctgcagttaagcaactttgaaatc-3', PstI site italics; pcaFD: 5 '-ggatgcattaaggatcaaaaaatgaaccctc- $3^{\prime}$ and $5^{\prime}$-ggatgcat ttaagcgaaatgctgtgc- $3^{\prime}$, NsiI site italics; pcaHGBC: $5^{\prime}$-aa ctgcagagacgcagaaaggtctc- 3 ' and $5^{\prime}$-ggctgcagttactgaaggtct gacac-3', NsiI site italics). The native ribosome binding site (RBS) was modified with the consensus RBS sequence 
(bold) for high expression. The amplified DNAs of pcalJ (1,404 bp), pcaFD (1,998 bp), and pcaHGBC (2,799 bp) were digested with PstI or NsiI, respectively, and were further ligated with PstI-digested pSL360 (same overhang with NsiI digestion) resulting in pSL360-pcaIJ, pSL360-pcaFD, and pSL360-pcaHGBC, respectively. The constructed vectors were electroporated (2 mm cuvette, $25 \mu \mathrm{F}, 200 \Omega, 2.5 \mathrm{kV})$ using an ECM 630 electroporation system (BTX, Holliston, MA, USA) into the wild-type $C$. glutamicum, after sequence verifications at a sequencing facility (Macrogen co., Seoul, Korea).

\section{Preparation of total RNA and RT-qPCR}

Total RNA was extracted from C. glutamicum cells using TRIzol $^{\odot}$ reagent (Invitrogen, Carlsbad, CA, USA) and the NucleoSpin ${ }^{\oplus}$ RNA II Kit (Macherey-Nagel, Düren, Germany) according to the manufacturer's instructions with the following modifications. C. glutamicum cells were harvested at an OD600 of 15, resuspended in TRIzol ${ }^{\odot}$ reagent, and transferred to vials containing glass beads (acid-washed, 212-300 $\mu \mathrm{m}$, Sigma-Aldrich, MO, USA). After cell disruption using Mini-Beadbeater-16 (Biospec, Bartlesville, PA, USA), the suspension was centrifuged, and the supernatant was applied to NucleoSpin ${ }^{\bullet}$ RNA II Kit (Macherey-Nagel, Düren, Germany). $50 \mathrm{ng}$ of total RNA of C. glutamicum cells were used to cDNA synthesis using ReverTra Ace- $\alpha-^{-}$(TOYOBO, Osaka, Japan) according to the manufacturer's instructions, respectively. THUNDERBIRD $^{\text {Ts }}$ SYBR $^{\circledR}$ qPCR Mix (TOYOBO, Osaka, Japan) and the Mx3005P QPCR System (Agilent Technologies, Santa Clara, CA, USA) were used for gene expression analysis. The RT-qPCR process was verified by melting curve and melting peak analyses. Relative quantity and standard error values from the expression analysis were calculated with MxPro-Mx3005P software ver. 4.10 (Agilent Technologies, Santa Clara, CA, USA). The following primers were used for detecting transcription level of pca genes: pcaI, 5'-acccagatgcagcaatga-3' and $5^{\prime}$-gacgcggttgacgtaaattc-3'; pcaJ, 5'-atcggcatgccta cacttatc- $3^{\prime}$ and $5^{\prime}$-gttcctcttcagttgggtaagg- $3^{\prime} ;$ pcaF, $5^{\prime}$-cc actgggttccggtattt- $3^{\prime}$ and $5^{\prime}$-gcgaaagcttcgttgagttc- $3^{\prime} ;$ pcaD, $5^{\prime}$-aacttccgacaacaccttgg- $3^{\prime}$ and $5^{\prime}$-cgatgacgcggaaatcct tat-3'; pcaH, 5'-ggaccgttatgccaggtaat- $3^{\prime}$ and $5^{\prime}$-ccgtaaa ctgacgaccatagag-3'; pcaG, 5'-cgctacgagcagtcgaatatc- $3^{\prime}$ and $55^{\prime}$-aaaccgatgtggacgtaagg- $3^{\prime} ;$ pcaB, $5^{\prime}$-ccgatctttatac tccgaccttg- $3^{\prime}$ and $5^{\prime}$-gcctccacgacaagaagatt- $3^{\prime} ;$ pcaC, $5^{\prime}$-tc gctatgaaaccggaatgaa- $3^{\prime}$ and $5^{\prime}$-cctgaaacttctcagtcacctc- $3^{\prime}$; $16 \mathrm{~S}$ rRNA, 5'-acccttgtcttatgttgccag- ${ }^{\prime}$ ' and $5^{\prime}$-tgtaccgac cattgtagcatg- $3^{\prime}$.

\section{Agar diffusion test}

The tolerance of C. glutamium strains against various oxidative stressors were estimated by the agar diffusion test. Cells in log phase were mixed with $0.7 \%$ agar solution, and the mixture $(3 \mathrm{~mL})$ was poured onto $1.6 \%$ bottom agar plate containing $20 \mathrm{~mL}$ of $\mathrm{BHI}$ medium (Bacto $^{\mathrm{TM}}$ Brain heart infusion $37 \mathrm{~g} / \mathrm{L}$, Cockeysville, MD, USA). A paper disc (6 mm diameter, Adventec, Tokyo, Japan) soaked with $20 \mu \mathrm{L}$ of oxidative stressor (14\% and $28 \% \mathrm{H}_{2} \mathrm{O}_{2}, 1 \mathrm{M}$ diamide, or $10 \%$ cumene hydroperoxide, respectively) was placed on top of the agar, and the plate was incubated at $30^{\circ} \mathrm{C}$ for $24 \mathrm{~h}$.

\section{Radical scavenging activity assay}

Free radical scavenging activity of cell extract was estimated using 2, 2-diphenyl-1-picrylhydrazyl (DPPH), that is a stable free radical and decolorized when acquire an electron (Afify et al. 2012). The bacterial cells grown to $\mathrm{OD}_{600 \mathrm{~nm}}=10$ in $\mathrm{BHI}$ medium were harvested $\left(5,000 \mathrm{rpm}\right.$ for $30 \mathrm{~min}$ at $\left.4^{\circ} \mathrm{C}\right)$ and disrupted by Mini-BeadBeater16 (BioSpec, Bartlesville, OK, USA) to prepare the cell free extract. The supernatant was mixed with the same volume of ethyl acetate. After vigorous mixing, the ethyl acetate layer was separated by centrifugation and filtrated by $0.22 \mu \mathrm{m}$ pore-membrane. The cell free extract was subjected to the free radical scavenging activity assay. Freshly prepared DPPH solution $(2.8 \mathrm{~mL})$ at a concentration of $5 \mathrm{mg} / 100 \mathrm{ml}$ (in ethanol) were mixed with the cell free extract $(200 \mu \mathrm{L})$ and incubated for $30 \mathrm{~min}$ in the dark at room temperature. Ethanol $(200 \mu \mathrm{L})$ was the control. The absorbance for the sample $\left(\mathrm{A}_{\text {sample }}\right)$ was monitored at $517 \mathrm{~nm}$ during incubation with 5 min intervals and further converted into the free radical scavenging activity according to the following equation:

$$
\begin{aligned}
& \text { ROS-scavenging activity }(\%) \\
& \quad=\left(\mathrm{A}_{\text {control }}-\mathrm{A}_{\text {sample }}\right) / \mathrm{A}_{\text {control }} \times 100
\end{aligned}
$$

The data were represented from the three biological repeated experiments.

\section{Results \\ Effect of pca gene clusters expressions on acquired $\mathrm{H}_{2} \mathrm{O}_{2}$-tolerance}

To determine whether the expression of pca gene clusters affects the growth of C. glutamicum under the oxidative stress conditions, cells were cultured in the MCGC minimal medium with or without $2 \mathrm{mM} \mathrm{H}_{2} \mathrm{O}_{2}$. The control strain (wild-type C. glutamicum carrying empty pSL360 vector) was unable to grow when $2 \mathrm{mM} \mathrm{H}_{2} \mathrm{O}_{2}$ was present whereas the strain was able to grow till O.D. $=18$ in $18 \mathrm{~h}$ without $\mathrm{H}_{2} \mathrm{O}_{2}$ (Figure 2). The wild-type strains carrying parts of pca gene clusters - pcaIJ, pcaFD, pcaHGBC were able to grow under the oxidative stress conditions. The expression of $p c a H G B C$ showed the most significant growth recovery (O.D. $=10.1$ at $30 \mathrm{~h}$ ), while the expression of pcalJ showed the least (O.D. = 2 at $30 \mathrm{~h}$ ). The expression of pcaFD showed an intermediate growth recovery (O.D. $=3.9$ at $30 \mathrm{~h}$ ). 

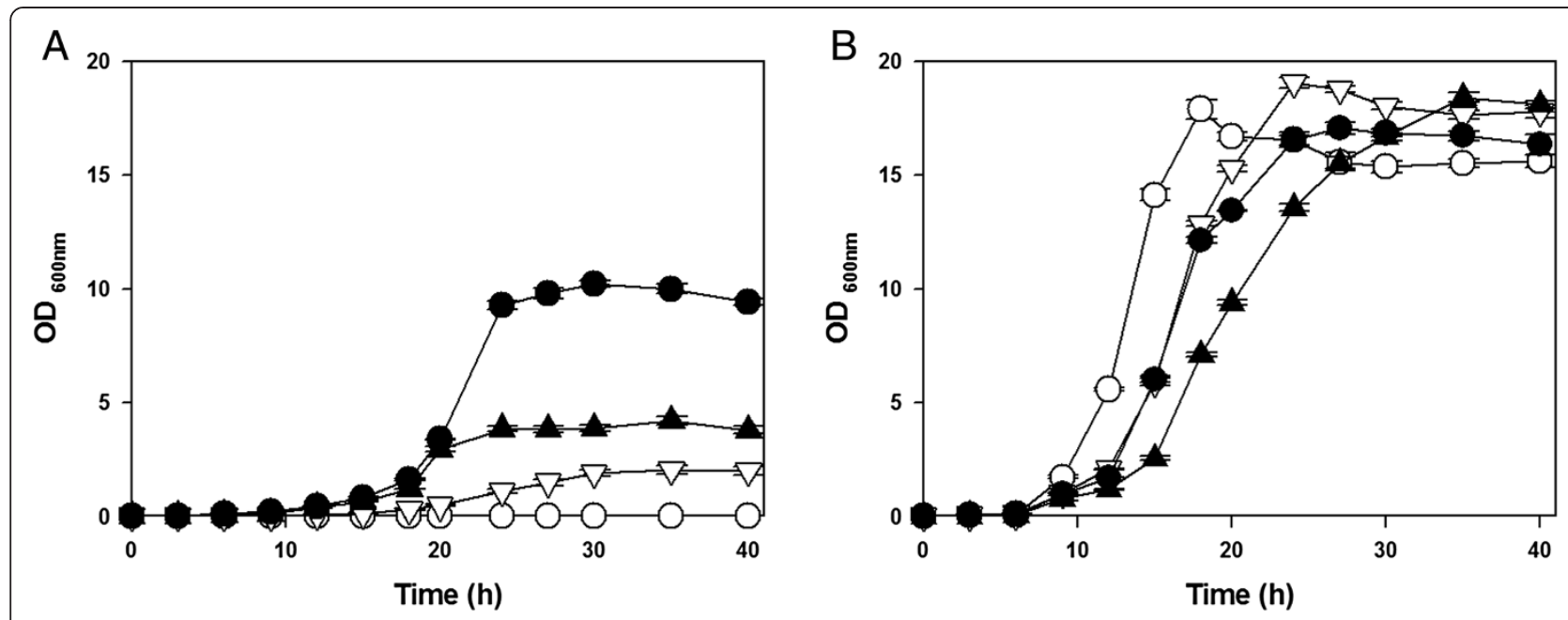

Figure 2 Growth of Corynebacterium glutamicum strains in minimal medium (A) with and (B) without $\mathrm{H}_{2} \mathrm{O}_{2}$ stress. Symbols are indicated as following: wild-type strain carrying empty vector (-०-); wild-type expressing pcalJ (- - -); wild-type expressing pcaFD (- $\mathbf{\Delta}-$ ); wild-type expressing pcaHGBC (- $\bullet$ ), respectively. Cells were cultured in MCGC minimal medium containing $25 \mu \mathrm{g} / \mathrm{mL}$ kanamycin, and the medium at the panel (A) additionally contained $2 \mathrm{mM} \mathrm{H}_{2} \mathrm{O}_{2}$.

For the verification of the expression of the pca gene clusters, the transcripts of the pca genes in the strains under no $\mathrm{H}_{2} \mathrm{O}_{2}$-stress conditions were analyzed by RT-qPCR (Table 1). All mRNAs of the pca genes those carried by the vector showed greater level than those in the wild-type. Only the fold of increase were varied depending on the clusters in the vector, that is, the mRNA levels of pcaI and pcaJ in the C. glutamicum (pSL360-pcaIJ) were 1.75- and 1.23-fold higher than those in the wild-type, mRNAs of pcaF and pcaD in the C. glutamicum (pSL360-pcaFD) were 40- and 42-fold higher, and mRNAs of pcaH, pcaG, pcaB, and pcaC in the C. glutamicum (pSL360-pcaHGBC) were 10.1-, 8.8-, 7.7-, 11.9-fold higher, respectively.
Effect of $p c a$ gene clusters expressions on the other oxidative stressors

To verify the effects of the expression of $p c a$ gene clusters on the tolerance against other oxidative stressors, agar diffusion tests were performed (Figure 3). The inhibition zones of the strain expressing $p c a$ gene clusters were smaller than that of the control against all tested oxidizing stressors (i.e., $14 \%$ and $28 \% \mathrm{H}_{2} \mathrm{O}_{2}, 1 \mathrm{M}$ diamide, and $10 \%$ cumene hydroperoxide). The size of inhibition zones were in good agreement with the growth properties, that is, the smallest inhibition zone against the oxidative stress was found in the pcaHGBC expressing strain and the largest in the pcaIJ expressing strain, though still more tolerant than the control strain.

Table 1 mRNA transcription levels of pca genes

\begin{tabular}{|c|c|c|c|c|c|}
\hline \multirow{2}{*}{$\begin{array}{l}\text { Target } \\
\text { gene }\end{array}$} & \multirow{2}{*}{$\frac{\text { RNA-seq }^{\text {a }} \text { (RPKM) }}{\text { WT }}$} & \multicolumn{4}{|c|}{$\mathrm{RT}^{\mathrm{qPCR}}{ }^{\mathrm{b}}$ (relative fold) } \\
\hline & & WT & + pcalJ & $+p c a F D$ & $+p c a H G B C$ \\
\hline pcal & 135.7 & $1.00 \pm 0.39$ & $1.75 \pm 0.36$ & $1.55 \pm 0.50$ & $0.61 \pm 0.17$ \\
\hline pcal & 134.8 & $1.00 \pm 0.15$ & $1.23 \pm 0.14$ & $2.56 \pm 0.27$ & $1.10 \pm 0.12$ \\
\hline pcaF & 220.9 & $1.00 \pm 0.05$ & $0.24 \pm 0.03$ & $40.2 \pm 6.6$ & $1.00 \pm 0.15$ \\
\hline$p c a D$ & 183.6 & $1.00 \pm 0.18$ & $0.25 \pm 0.05$ & $42.5 \pm 5.9$ & $1.00 \pm 0.13$ \\
\hline pcaH & 172.0 & $1.00 \pm 0.02$ & $0.23 \pm 0.01$ & $2.61 \pm 0.12$ & $10.1 \pm 0.95$ \\
\hline$p c a G$ & 116.7 & $1.00 \pm 0.01$ & $0.23 \pm 0.02$ & $2.45 \pm 0.16$ & $8.83 \pm 0.60$ \\
\hline$p c a B$ & 217.5 & $1.00 \pm 0.22$ & $0.19 \pm 0.04$ & $2.31 \pm 0.39$ & $7.72 \pm 0.42$ \\
\hline pcaC & 77.7 & $1.00 \pm 0.02$ & $0.21 \pm 0.02$ & $2.52 \pm 0.23$ & $11.9 \pm 0.71$ \\
\hline
\end{tabular}

The cells were grown in MCGC minimal medium without $\mathrm{H}_{2} \mathrm{O}_{2}$-stress and harvested in the log phase for mRNA preparation.

${ }^{a}$ Adapted from (Lee et al. 2013b) (NCBI Gene Expression Omnibus access code: GSE41232) RPKM (reads per kilo base per million).

${ }^{\mathrm{b}}$ This study. Values are mean \pm SD from three independent experiments. 


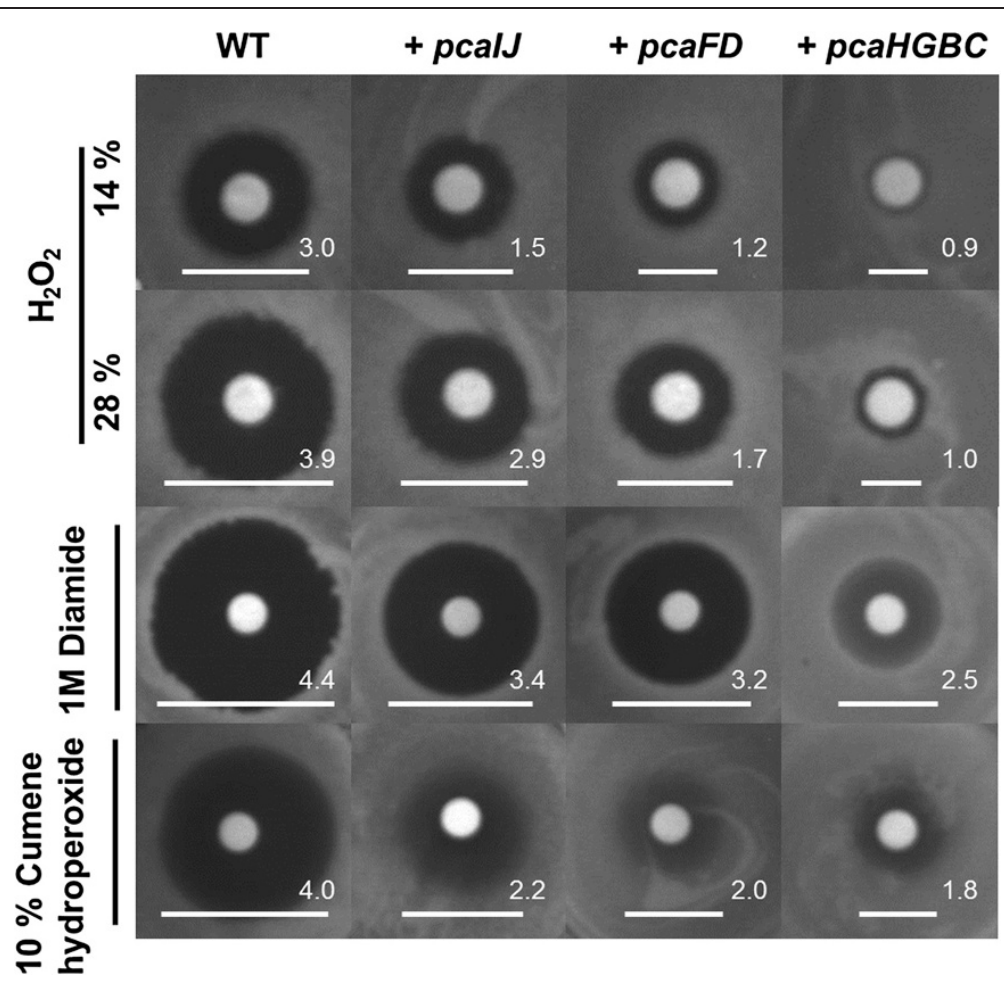

Figure 3 Growth inhibitions of Corynebacterium glutamicum strains against oxidative stressors. WT: C. glutamicum wild-type strains carrying empty vector, $+p c a l$ : wild-type expressing pcals; + pcaFD: wild-type expressing pcaFD; + pcaHGBC: wild-type expressing pcaHGBC. Vertical bars indicate the kind of oxidative stressors on the each paper disc. The agar plate contained BHI medium and a paper disc contained $20 \mu \mathrm{L}$ of each stressor. The white bars and numbers indicate the size of each inhibition zone in centimeters.

\section{Effect of pca gene clusters expressions on the intracellular ROS-scavenging activity}

To understand the reason of the acquired oxidative stress-tolerance in the $p c a$ gene clusters expressing $C$. glutamicum strains, ROS-scavenging activity of the cell extract was estimated by DPPH assay (Figure 4 and Additional file 1: Figure S1). The cell extract from the pcaHGBC expressing strain showed 3-times greater ROSscavenging activity $(47.7 \pm 1.6 \%)$ than that from the wild-type $(16.4 \pm 1.1 \%)$. The ROS-scavenging activities of the cell extracts from the pcaFD and pcalJ expressing strains were $39.1 \pm 2.3 \%$ and $30.9 \pm 1.4 \%$, respectively.

\section{Discussion}

Constitutive overexpressions of pca gene clusters enabled wild-type $C$. glutamicum to tolerate oxidizing stressors, and pcaHGBC expression was the most effective among pca gene clusters (Figures 2 and 3). The ROS-scavenging activity of the cell extract was enhanced by the pca gene clusters expressions (Figure 4). Considering that the $\beta$-ketoadipate pathway by the $p c a$ gene clusters is involved in the degradations of aromatic intermediates (protocatechuate [PCA, 3,4-dihydroxybenzoate], vanillate [3-methoxy,4-hydroxybenzoate], and 4-hydroxybenzoate) and those were also found in natural herbs as antioxidants
(Zheng and Wang 2001), it would be reasonable to estimate that aromatic intermediates have been synthesized and contributed to ROS-scavenging activity to tolerate the oxidative stresses (Additional file 2: Figure S3).

The wild-type $C$. glutamicum transcribed the $p c a$ gene clusters at a low level, even though no aromatic carbon sources were present in the medium (NCBI Gene Expression Omnibus access code: GSE41232), and the transcription level of pcaC was the lowest among the pca genes: pcaI: 135.7; pcaJ: 134.8; pcaF: 220.9; pcaD: 183.6; pcaG: 116.7; pcaH:172.0; pcaB: 217.5; pcaC: 77.7 RPKM (reads per kilo base per million), respectively (Lee et al. 2013b). This suggested pcaC, a putative 4-carboxymuconolactone decarboxylase, might have been the bottleneck step for synthesis of aromatic antioxidants in the wild-type strain, and overexpression of pcaHGBC might have been mainly responsible for the bottleneck of the pathway among all pca gene cluster expressions. Table 1 showed the mRNA levels of $p c a F, p c a D, p c a H$, pcaG, pcaB, and pcaC in the C. glutamicum (pSL360pcaIJ) were even lower $(0.19-\sim 0.25$-fold) than those in the wild-type, and this might be the reason why the pcalJ cluster expression showed the least effect of oxidative stress resistance among the tested $p c a$ gene clusters. 


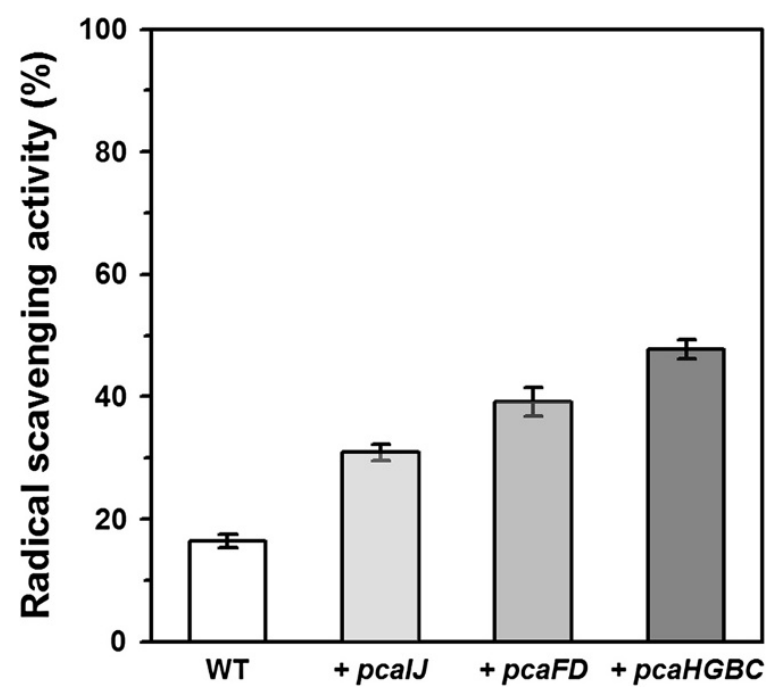

Figure 4 ROS-scavenging activities of the cell free extracts from Corynebacterium glutamicum strains. The cell extracts from the C. glutamicum wild-type strains carrying empty vector, pcall-expressing vector, pcaFD-expressing vector, and pcaHGBC-expressing vector are indicated as WT, $+p c a l J,+p c a F D$, and $+p c a H G B C$, respectively. Cells were harvested at $\mathrm{OD}=10$ in the $\mathrm{BH}$ medium for cell extract preparations.

It is not clear the artificial pca gene clusters expressions have led the actual intracellular accumulation of aromatic intermediates. We were not able to detect the actual accumulation of recognizable aromatic metabolites from the methanolic extracts of the recombinant strains based on GC/MS analysis (Additional file 1: Figure S2), though few metabolites have been changed between the wildtype and the recombinant strains. The ROS-scavenging aromatic intermediates might not have been preserved enough to be detected. Okada and Okada reported that the supplementation of aromatic compounds in methanolic extract derived from broad bean increased the growth rate of human fibroblasts cells by ROS-scavenging activity (Okada and Okada 2007), and the addition of $50 \mathrm{mg} / \mathrm{L}$ of a mixture of phenol carboxylic acids derived from wine (caffeate, ferulate, $p$-coumarate, gallate) was reported to stimulate bacterial growth (Rozes et al. 2003). The addition of $0.1 \%$ gallate was also reported to enhance the aerobic growths of Escherichia coli ATCC 11775 and Staphylococcus enteridis ATCC 13076 1.5-fold and 2-fold, respectively (Lee et al. 2006). These reports implied that an overexpression of the aromatic compound-synthetic pathway might be beneficial for the improved growth rates of industrial strains, considering the facts that cellular damages from ROS in aerobic bioprocess are unavoidable, and that aromatic compounds are able to scavenge growth-harmful ROS.

A number of microorganisms have been reported to produce aromatic compounds and their derivatives, via the aromatic compound-degrading $\beta$-ketoadipate pathway (Harwood and Parales 1996). The biologically-beneficial properties of aromatic compounds as anti-oxidant, anti- cancer, and anti-inflammatory compounds have encouraged their synthesis using microorganisms. Advances in metabolic engineering and synthetic biology enabled the artificial biosynthesis of aromatic compounds (e.g. anthocyanins, caffeic acid, coumaric acid, hydroxybenzoic acid, ferulic acid, and genisteinin) using E. coli and S. cerevisiae (Yan et al. 2005; Katsuyama et al. 2007; Lin and Yan 2012; Kang et al. 2012). C. glutamicum has been reported to degrade aromatic compounds by $\beta$-ketoadipate pathway (Shen et al. 2004; Shen and Liu 2005; Merkens et al. 2005; Brinkrolf et al. 2006), and their regulations have been studied (Qi et al. 2007; Haußmann et al. 2009; Haußmann and Poetsch 2012). Despite the industrial importance of $C$. glutamicum, there has been no report of the production of aromatic compounds from C. glutamicum. The findings in this study suggest that C. glutamicum is a potentially suitable host for the production of aromatic antioxidants via the $\beta$-ketoadipate pathway, as well as being suitable for further applications as an oxidative stress-tolerant host. Introduction of $\beta$-ketoadipate pathway of C. glutamicum into other species might be another application. The authors recently found the engineered Escherichia coli harboring greater intracellular ATP, even though useful for application (Kim et al. 2012; Kim et al. 2011), showed a growth defect (Lee et al. 2013a) and intracellular accumulation of ROS was suspected as the reason of growth inhibition. The ROS scavenging activities from the pca gene clusters might enabled the engineered $E$. coli to reduce the ROS from the high ATP and to lead growth recovery.

In conclusion, the wild-type C. glutamicum acquired oxidative stress-tolerance based on the increased ROSscavenging activity by introducing the $\beta$-ketoadipate path- 
way gene clusters, which suggests that the intermediates of the $\beta$-ketoadipate pathway contributed to the acquired tolerance. This finding could be further applied to develop a synthetic cell which is oxidative stress-tolerant and rapid growing industrial strain under oxidative stress conditions.

\section{Additional files}

Additional file 1: Figure $\mathbf{S 1}$. Kinetics of DPPH radical scavenging activity of cell free extracts of Corynebacterium glutamicum. Figure S2. The GC chromatogram of the methanolic extracts of $C$. glutamicum strains.

Additional file 2: Figure S3. Summary for the expression effect of pca gene clusters on the artificial oxidative stress-tolerance in Corynebacterium glutamicum.

\section{Competing interest}

The authors declare that they have no competing interests.

\section{Acknowledgements}

This study was financially supported by the Korean Ministry of Science, ICT \& Future Planning (Intelligent Synthetic Biology Center of Global Frontier Project 2012M3A6A8054887). Kim P was supported by the 2013 research fellowship from the Catholic University of Korea. The authors extend their appreciation to Dr. Lee CH (Metamass Co. and Konkuk University) for supporting GC-TOF-MS analysis.

\section{Author details}

'Department of Biotechnology, The Catholic University of Korea, Bucheon, Gyeonggi 420-743, Korea. ${ }^{2}$ Department of Biological Engineering, Inha University, Inchon 402-751, Korea. ${ }^{3}$ Department of Biotechnology and Bioinformatics, Korea University, Jochiwon, Chungnam 339-700, Korea. ${ }^{4}$ Present address: ST Pharm Co., Siheung, Gyeonggi 429-912, Korea.

Received: 4 January 2014 Accepted: 8 February 2014

Published online: 18 March 2014

\section{References}

Afify AEMR, Romeliah RM, Sultan SIM, Hussein MM (2012) Antioxidant activity and biological evaluations of probiotic bacteria strains. Int J Acad Res 4(6):131-139. doi:10.7813/2075-4124.2012/4-6

Brinkrolf K, Brune I, Tauch A (2006) Transcriptional regulation of catabolic pathways for aromatic compounds in Corynebacterium glutamicum. Genet Mol Res 5(4):773-789

Eggeling L, Bott M (2005) Handbook of Corynebacterium glutamicum. CRC press (Talyor \& Francis Group), Boca Raton, FL

Fridovich I (1998) Oxygen toxicity: a radical explanation. J Experiment Biol 201(8):1203-1209

Harwood CS, Parales RE (1996) The $\beta$-ketoadipate pathway and the biology of self-identity. Ann Rev Microbiol 50(1):553-590

Haußmann U, Poetsch A (2012) Global proteome survey of protocatechuate- and glucose-grown Corynebacterium glutamicum reveals multiple physiological differences. J Proteomics 75(9):2649-2659

Haußmann U, Qi S-W, Wolters D, Rögner M, Liu S-J, Poetsch A (2009) Physiological adaptation of Corynebacterium glutamicum to benzoate as alternative carbon source - a membrane proteome-centric view. Proteomics 9(14):3635-3651

Kang SY, Choi O, Lee JK, Hwang BY, Uhm TB, Hong YS (2012) Artificial biosynthesis of phenylpropanoic acids in a tyrosine overproducing Escherichia coli strain. Microb Cell Fact 11:153. doi:10.1186/1475-2859-11-153

Katsuyama Y, Miyahisa I, Funa N, Horinouchi S (2007) One-pot synthesis of genistein from tyrosine by coincubation of genetically engineered Escherichia coli and Saccharomyces cerevisiae cells. Appl Microbiol Biotechnol 73(5):1143-1149. doi:10.1007/s00253-006-0568-2

Kim HJ, Kwon YD, Lee SY, Kim P (2012) An engineered Escherichia coli having a high intracellular level of ATP and enhanced recombinant protein production. Appl Microbiol Biotechnol 94(4):1079-1086. doi:10.1007/ s00253-011-3779-0

Kim S, Lee CH, Nam SW, Kim P (2011) Alteration of reducing powers in an isogenic phosphoglucose isomerase (pgi)-disrupted Escherichia coli expressing NAD(P)-dependent malic enzymes and NADP-dependent glyceraldehyde 3-phosphate dehydrogenase. Lett Appl Microbiol 52(5):433-440. doi:10.1111/j.1472-765X.2011.03013.x

Lee HC, Jenner AM, Low CS, Lee YK (2006) Effect of tea phenolics and their aromatic fecal bacterial metabolites on intestinal microbiota. Res Microbiol 157(9):876-884. doi:10.1016/j.resmic.2006.07.00

Lee HJ, Kim HJ, Seo J, Na YA, Lee J, Lee JY, Kim P (2013a) Estimation of phosphoenolpyruvate carboxylation mediated by phosphoenolpyruvate carboxykinase (PCK) in engineered Escherichia coli having high ATP. Enzyme Microb Technol 53(1):13-17. doi:10.1016/j.enzmictec.2013.04.001

Lee JY, Seo J, Kim ES, Lee HS, Kim P (2013b) Adaptive evolution of Corynebacterium glutamicum resistant to oxidative stress and its global gene expression profiling. Biotechnol Lett 35(5):709-717. doi:10.1007/s10529-012-1135-9

Li Q, Harvey LM, McNeil B (2009) Oxidative stress in industrial fungi. Crit Rev Biotechnol 29(3):199-213

Lin Y, Yan Y (2012) Biosynthesis of caffeic acid in Escherichia coli using its endogenous hydroxylase complex. Microb Cell Fact 11:42. doi:10.1186/1475-2859-11-42

Merkens H, Beckers G, Wirtz A, Burkovski A (2005) Vanillate Metabolism in Corynebacterium glutamicum. Curr Microbiol 51(1):59-65

Okada M, Okada Y (2007) Effects of methanolic extracts from broad beans on cellular growth and antioxidant enzyme activity. Environ Health Prev Med 12(6):251-257. doi:10.1007/BF02898032

Park SD, Lee SN, Park IH, Choi JS, Jeong WK, Kim Y, Lee HS (2004) Isolation and Characterization of Transcriptional Elements from Corynebacterium glutamicum. J Microbiol Biotechnol 14(4):789-795

Qi S-W, Chaudhry MT, Zhang Y, Meng B, Huang Y, Zhao KX, Poetsch A, Jiang C-Y, Liu S, Liu S-J (2007) Comparative proteomes of Corynebacterium glutamicum grown on aromatic compounds revealed novel proteins involved in aromatic degradation and a clear link between aromatic catabolism and gluconeogenesis via fructose-1,6-bisphosphatase. Proteomics 7(20):3775-3787

Rozes N, Arola L, Bordons A (2003) Effect of phenolic compounds on the co-metabolism of citric acid and sugars by Oenococcus oeni from wine. Lett Appl Microbiol 36(5):337-341

Shen X-H, Liu Z-P, Liu S-J (2004) Functional identification of the gene locus (ncg12319 and characterization of catechol 1,2-dioxygenase in Corynebacterium glutamicum. Biotechnol Lett 26(7):575-580

Shen X-H, Zhou N-Y, Liu S-J (2012) Degradation and assimilation of aromatic compounds by Corynebacterium glutamicum: another potential for applications for this bacterium? Appl Microbiol Biotechnol 95(1):77-89

Shen X, Liu S (2005) Key enzymes of the protocatechuate branch of the $\beta$-ketoadipate pathway for aromatic degradation in Corynebacterium glutamicum. Science in China Series C. Life Sci 48(3):241-249

von der Osten CH, Gioannetti C, Sinskey AJ (1989) Design of a defined medium for growth of Corynebacterium glutamicum in which citrate facilitates iron uptake. Biotechnol Lett 11(1):11-16

Yan Y, Chemler J, Huang L, Martens S, Koffas MA (2005) Metabolic engineering of anthocyanin biosynthesis in Escherichia coli. Appl Environ Microbiol 71(7):3617-3623. doi:10.1128/AEM.71.7.3617-3623.2005

Zhao KX, Huang Y, Chen X, Wang NX, Liu SJ (2010) PcaO positively regulates pcaHG of the beta-ketoadipate pathway in Corynebacterium glutamicum. J Bacteriol 192(6):1565-1572. doi:10.1128/JB.01338-09

Zheng W, Wang SY (2001) Antioxidant activity and phenolic compounds in selected herbs. J Agric Food Chem 49(11):5165-5170

doi:10.1186/s13568-014-0015-1

Cite this article as: Lee et al:: Artificial oxidative stress-tolerant

Corynebacterium glutamicum. AMB Express 2014 4:15. 